\title{
Sparse Beamforming for Limited-Backhaul Network MIMO System via Reweighted Power Minimization
}

\author{
Binbin Dai and Wei Yu \\ Department of Electrical and Computer Engineering \\ University of Toronto, Toronto, Ontario M5S 3G4, Canada \\ Emails: \{bdai,weiyu\}@comm.utoronto.ca
}

\begin{abstract}
This paper considers a downlink multicell cooperation model in which the base-stations (BSs) are connected to a central processor $(\mathrm{CP})$ via rate-limited backhaul links. A usercentric clustering model is adopted where each scheduled user is cooperatively served by a cluster of BSs, and the serving BSs for different users may overlap. This paper formulates an optimal joint clustering and beamforming design problem in which each user dynamically forms a sparse network-wide beamforming vector whose non-zero entries correspond to the serving BSs. Specifically, we assume a fixed signal-to-interference-and-noise ratio (SINR) constraint for each user, and investigate the optimal tradeoff between the sum transmit power and the sum backhaul capacity needed to form the cooperating clusters. Intuitively, larger cooperation size leads to lower transmit power, because interference can be mitigated through cooperation, but it also leads to higher sum backhaul, because user data needs to be made available to more BSs. Motivated by the compressive sensing literature, this paper formulates the sparse beamforming problem as an $\ell_{0}$-norm optimization problem, then uses the iterative reweighted $\ell_{1}$ heuristic to find a solution. A key observation of this paper is that the reweighting can be done on the $\ell_{2}$-norm square of the beamformers (i.e., the power) at the BSs. This gives rise to a weighted power minimization problem over the entire network, which can be solved using the uplink-downlink duality technique with low computational complexity. This paper further proposes judicious choice of the weights, and shows that the new algorithm can provide a better tradeoff between the sum power and the sum backhaul capacity in the high SINR regime than previous algorithms.
\end{abstract}

\section{INTRODUCTION}

Wireless cellular networks are increasingly deployed with progressively smaller cell sizes in order to support the ever increasing demand for high-speed data. As a consequence, intercell interference is now the main physical-layer bottleneck in cellular networks. Multicell cooperation, which allows neighboring base-stations (BSs) to cooperate with each other for joint precoding and joint processing of user data, is a promising technology for intercell interference mitigation [1]. This emerging architecture, known as network multiple-input multiple-output (MIMO) [2], has the potential to significantly improve the overall throughput of the cellular network.

The idealized implementation of multicell cooperation, where all BSs in the entire network cooperate and share the data for all users, is clearly impractical. One way to implement multicell cooperation in practice is to connect all the BSs with a central processor $(\mathrm{CP})$ via rate-limited backhaul links. For downlink transmission, the $\mathrm{CP}$ then only needs to distribute the user's data to its serving BSs. Although fixed clustering scheme, which simply groups the neighboring BSs into a larger cluster, has already shown reasonable performance gain, in such a scheme users at the cluster edge still suffer from considerable intercell interference which limits the benefit of network MIMO [3]. This paper adopts an alternative model, called user-centric clustering, where the BS clusters are not fixed but are determined for each user individually. In this model, each user dynamically selects a set of favorable BSs; these BSs then cooperatively serve the user using joint precoding techniques. The benefit of user-centric clustering is that it has no explicit cluster edges.

This paper addresses the question of how to determine the best serving set of BSs and how to design the best networkwide beamformer for each user. This is not a straightforward task, because from the users' perspective, each user wishes to be served cooperatively by as many BSs as possible, while from the BSs' perspective, serving more users consumes more power and backhaul capacity. There exists therefore a tradeoff between the user rates, the transmit powers, and the backhaul capacity. Further, the beamformer design problem for the network MIMO system with user-centric clustering is also nontrivial, because the sets of BSs serving different users may overlap. The traditional zero-forcing (ZF) and minimum mean square error (MMSE) beamforming designs specifically developed for the single cell case need to be generalized [4].

This paper proposes a sparse beamforming design to solve the above problem. Specifically, we design a network-wide sparse beamforming vector for each user, where the nonzero entries correspond to the serving BSs. We formulate the optimization problem as that of finding the optimal tradeoff between the total transmit power and the sum backhaul capacity over all BSs, under fixed signal-to-interference-andnoise (SINR) constraints at the remote users. Intuitively, higher backhaul capacity allows more BSs to cooperate, which leads to less inter-cluster interference and therefore less transmit power that would be needed to serve the users at the fixed SINRs.

The above optimization problem can be formulated as an $\ell_{0}$-norm optimization problem. But, such a formulation is nonconvex. Motivated by the compressive sensing literature [5], this paper uses an iterative reweighted $\ell_{1}$ technique and proposes to approximate the backhaul rates in terms of the weighted $\ell_{2}$-norm square of the beamfomers. The key observation here is that this transforms the problem into a 
weighted power minimization problem with SINR constraints, which can then be solved using the uplink-downlink duality approach with low computational complexity.

The sparse beamforming problem has already been addressed previously in the literature [6]-[8]. In [6], the authors use the $\ell_{1}$-norm of the beamforming vector to approximate the cluster size, while [8] proposes an algorithm to improve the $\ell_{1}$ norm approximation by reweighting. In both cases, the cluster size is determined from the $\ell_{2}$-norm of the beamformers at each BS, and the resulting optimization problem becomes a second-order cone programming (SOCP) problem [9], which can be solved numerically by the interior-point method. To reduce the computational complexity of the interior-point method, the authors of [8] further propose a second algorithm, which first solves the sum power minimization problem, then iteratively removes the links corresponding to the smallest link transmit power. One of the main contributions of this paper is to show that by working with the weighted $\ell_{2}$-normsquare of the beamformers (which is equivalent to power), instead of the $\ell_{2}$-norm itself, we can formulate the problem as a weighted power minimization for which the well-known uplink-downlink duality approach can be used to solve the problem efficiently. Our algorithm can therefore be thought of as combining iterative reweighting with the low-complexity feature of the weighted power minimization formulation.

Further, this paper adopts a new weight updating rule in $\ell_{1}$ reweighting, which is different from the weight updating method of [8], where the weights are chosen as inversely proportional to the beamformer entries. The new weight updating rule enables the proposed algorithm to achieve a better tradeoff between the sum power and the sum backhaul capacity, as compared to the existing algorithms in [8].

The idea of compressive sensing has been used in various scenarios in communication system design. In [10], the authors design uplink sparse MMSE receivers for the uplink multicell cooperation model using the $\ell_{1}$-norm approximation, while [11] uses similar ideas for joint power and link admission control in an interference channel. The problem setup of this paper is related to [12], which investigates the joint clustering and beamforming design for the downlink by adding a $\ell_{2}$-norm approximation of the cluster size for each user as a penalized item. However, [12] differs from the present paper by focusing on the weighted sum rate maximization problem, instead of power minimization. This allows [12] to use a weighted MMSE technique to find a local optimal solution to the rate maximization problem. Finally, the problem setup of the present paper is also related to [13], which proposes a heuristic way of solving the problem of clustering, beamforming design, user scheduling, and power allocation in a decoupled fashion.

\section{System Model and Problem Formulation}

Consider a network MIMO system with $L$ BSs connected to a $\mathrm{CP}$ via limited backhaul links with a total capacity limit $C$, as depicted in Fig. 1. Suppose that there are $K$ single antenna users, and the $\mathrm{CP}$ has access to all user data and channel state information (CSI) in the system. Although a fully

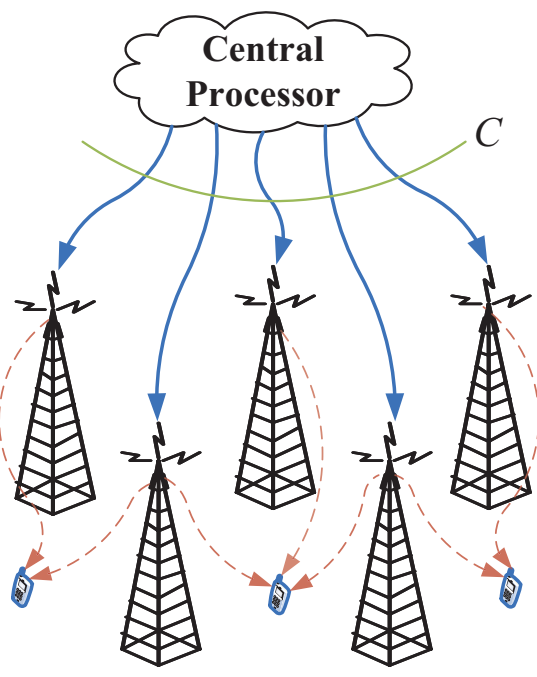

Fig. 1. Downlink multicell cooperation system model with all the BSs connected to a $\mathrm{CP}$ via limited backhaul links under a total capacity limit $C$, where each scheduled user is cooperatively served by a potentially overlapping subset of BSs.

cooperative network MIMO system, where every single user is served by all the $L$ BS's, can dramatically reduce the intercell interference, it also requires very high backhaul capacity, because the $\mathrm{CP}$ needs to make every user's data available at every BS. This paper considers a more practical architecture in which each user selects only a subset of serving BSs (which are potentially overlapping) and the $\mathrm{CP}$ only distributes the user's data to its serving BSs.

Let $\mathbf{w}_{k} \in \mathbb{C}^{M L \times 1}=\left[\mathbf{w}_{k}^{1}, \mathbf{w}_{k}^{2}, \cdots, \mathbf{w}_{k}^{L}\right]$ be the transmit beamformer over all BSs for user $k$, where $\mathbf{w}_{k}^{l} \in \mathbb{C}^{M \times 1}$ is the transmit beamformer from BS $l(l=1,2, \cdots, L)$ to user $k(k=1,2, \cdots, K)$. Note that $\mathbf{w}_{k}^{l}=\mathbf{0}$ if $\mathrm{BS} l$ is not part of user $k$ 's serving cluster. The received signal $y_{k} \in \mathbb{C}$ at user $k$ can be written as:

$$
y_{k}=\mathbf{h}_{k}^{H} \mathbf{w}_{k} s_{k}+\sum_{j \neq k}^{K} \mathbf{h}_{k}^{H} \mathbf{w}_{j} s_{j}+n_{k}
$$

where $\mathbf{h}_{k} \in \mathbb{C}^{M L \times 1}$ denotes the CSI vector from all the BSs to user $k, s_{k} \sim \mathcal{C N}(0,1)$ and $n_{k} \sim \mathcal{C N}\left(0, \sigma^{2}\right)$ are the intended signal and the receiver noise for user $k$, respectively.

The SINR for user $k$ can be expressed as:

$$
\operatorname{SINR}_{k}=\frac{\left|\mathbf{h}_{k}^{H} \mathbf{w}_{k}\right|^{2}}{\sum_{j \neq k}\left|\mathbf{h}_{k}^{H} \mathbf{w}_{j}\right|^{2}+\sigma^{2}} .
$$

The achievable rate for user $k$ is then

$$
R_{k}=\log \left(1+\operatorname{SINR}_{k}\right)
$$

Since each user's data only needs to be made available at its serving BSs, the sum backhaul capacity consumption $C_{k}$ needed for serving user $k$ can be represented as

$$
C_{k}=\left\|\left[\left\|\mathbf{w}_{k}^{1}\right\|_{2},\left\|\mathbf{w}_{k}^{2}\right\|_{2}, \cdots,\left\|\mathbf{w}_{k}^{L}\right\|_{2}\right]\right\|_{0} R_{k}
$$


where $\|\cdot\|_{0}$ denotes the $\ell_{0}$-norm of a vector ${ }^{1}$, i.e. the number of nonzero entries in the vector.

We can now formulate an optimization problem that relates various network resources and the system throughput. The network resources considered in this paper consist of the backhaul capacities and the transmit powers at the BSs. Clearly more resources lead to higher throughput. But at fixed user throughput, there is also a tradeoff between the backhaul capacity and the transmit power. Intuitively, higher backhaul capacity allows for more BSs to cooperate, which leads to less interference; hence less transmit power is needed to achieve a target user rate.

Formally, this paper formulates the tradeoff between the total transmit power and the sum backhaul capacity over all BSs under fixed user data rates as the following optimization problem:

$$
\begin{gathered}
\underset{\mathbf{w}_{k}^{l}}{\operatorname{minimize}} \quad \sum_{k}\left\|\left[\left\|\mathbf{w}_{k}^{1}\right\|_{2},\left\|\mathbf{w}_{k}^{2}\right\|_{2}, \cdots,\left\|\mathbf{w}_{k}^{L}\right\|_{2}\right]\right\|_{0} R_{k} \\
+\eta \sum_{k} \sum_{l}\left\|\mathbf{w}_{k}^{l}\right\|_{2}^{2}
\end{gathered}
$$$$
\text { subject to } \operatorname{SINR}_{k} \geq \gamma_{k}, \forall k
$$

where $\eta \geq 0$ is a constant indicating the tradeoff between sum backhaul capacity and sum power, $\gamma_{k}$ is the SINR target for user $k$ and $R_{k}=\log _{2}\left(1+\gamma_{k}\right)$. This paper focuses on the numerical solution to this problem.

We note that the above problem formulation is not the only possibility here. For example, [12] studies the tradeoff between the user rates and the cluster size in a weighted sum rate maximization problem under fixed power constraints. As a further note, this paper considers the sum power and sum backhaul capacity only, but in practice the per-BS transmit power and the per-BS backhaul capacity are also of interest. We defer such studies to future work.

\section{Sparse BeAmforming Design Algorithms}

The optimization problem (5) is nonconvex due to the $\ell_{0}$ norm representation of the backhaul rate. Finding the global optimal solution to (5) is difficult. Motivated by the compressive sensing literature, we propose to solve (5) heuristically by iteratively relaxing the $\ell_{0}$-norm as a weighted $\ell_{1}$-norm. In this section, we first introduce our proposed algorithm and then compare it with existing algorithms.

\section{A. Proposed Algorithm with Reweighted Power Minimization}

First, we make an observation that if the $\ell_{2}$-norm in (4) is replaced by $\ell_{2}$-norm square, the overall $\ell_{0}$-norm remains the same. Thus, the backhaul consumption $C_{k}$ can also be written as

$$
C_{k}=\left\|\left[\left\|\mathbf{w}_{k}^{1}\right\|_{2}^{2},\left\|\mathbf{w}_{k}^{2}\right\|_{2}^{2}, \cdots,\left\|\mathbf{w}_{k}^{L}\right\|_{2}^{2}\right]\right\|_{0} R_{k}
$$

The basic idea of $\ell_{1}$-heuristics in compressive sensing is to replace the $\|\cdot\|_{0}$ norm by $\|\cdot\|_{1}$ norm in the optimization problem. Applying this idea to (6) and further introducing

\footnotetext{
${ }^{1}$ Strictly speaking, $\|\cdot\|_{0}$ is not a norm by definition since it does not satisfy the homogenous property. However, we still call it $\ell_{0}$-norm by convention.
}

the appropriate weights, $C_{k}$ can now be approximated as the weighted $\ell_{2}$-norm square of the beamformers, and the problem (5) can now be relaxed as

$$
\underset{\mathbf{w}_{k}^{l}}{\operatorname{minimize}} \sum_{k}\left(\sum_{l} \rho_{k}^{l}\left\|\mathbf{w}_{k}^{l}\right\|_{2}^{2}\right) R_{k}+\eta \sum_{k} \sum_{l}\left\|\mathbf{w}_{k}^{l}\right\|_{2}^{2}
$$

subject to $\operatorname{SINR}_{k} \geq \gamma_{k}, \forall k$

where $\rho_{k}^{l}$ is the weight associated with BS $l$ and user $k$.

Observe that the problem (7) can be further rearranged into the following form

$$
\begin{array}{ll}
\underset{\mathbf{w}_{k}^{l}}{\operatorname{minimize}} & \sum_{k, l} \alpha_{k}^{l}\left\|\mathbf{w}_{k}^{l}\right\|_{2}^{2} \\
\text { subject to } & \operatorname{SINR}_{k} \geq \gamma_{k}, \forall k
\end{array}
$$

where $\alpha_{k}^{l}=\rho_{k}^{l} R_{k}+\eta$. Since the $\ell_{2}$-norm square of the beamforming vectors are just the transmit powers at the BSs, the above optimization problem is just a weighted power minimization problem.

The weighted power minimization problem (8) has been extensively studied in the literature. The key point is that it can be solved efficiently using the well-known uplinkdownlink duality approach. The main novelty of this paper is thus the observation that this particular relaxation of $C_{k}$ as weighted $\ell_{2}$-norm square results in a problem formulation whose structure can be efficiently exploited by numerical algorithms.

Uplink-downlink duality for weighted power minimization is developed for single cell case in [14] and subsequently generalized to the multicell setting in [15]. This paper further generalizes duality to the case where the weight associated with each BS-user pair may be different.

Note that the solution to (8) for a fixed weight $\rho_{k}^{l}$ does not necessarily provide sufficient sparsity. However, by iteratively updating the weights $\rho_{k}^{l}$ and by solving problem (8) repeatedly with updated $\rho_{k}^{l}$, we eventually get a sparse network-wide beamforming vector for each user, where entries corresponding to the BSs outside of the optimal serving cluster go to zero in the limit. In this paper, we adopt the following reweighting function to update $\rho_{k}^{l}$

$$
\rho_{k}^{l}=\frac{1}{\left\|\mathbf{w}_{k}^{l}\right\|_{2}^{2 p}+\epsilon^{p}}
$$

where $p$ is some positive exponent and $\epsilon$ is adaptively chosen to be $\epsilon=\max \left\{\left(\min _{k, l}\left\|\mathbf{w}_{k}^{l}\right\|_{2}^{2}\right), \tau\right\}$ and $\tau$ is some small positive value, and $\mathbf{w}_{k}^{l}$ is the beamforming vector from the previous iteration. We show numerically that with properly chosen $p$, the reweighting function (9) improves upon the performance of previous algorithms.

To completely characterize the proposed algorithm, we still need to give the solution to (8) based on the following generalization of uplink-downlink duality.

Proposition 3.1: The downlink weighted power minimization problem (8) is equivalent to the following uplink sum power minimization problem in the sense that they have 
the same optimal solution up to a scalar factor, i.e., $\mathbf{w}_{k}=$ $\sqrt{\delta_{k}} \hat{\mathbf{w}}_{k}, \forall k$ :

$$
\begin{aligned}
\underset{\lambda_{k}, \hat{\mathbf{w}}_{k}}{\operatorname{minimize}} & \sum_{k} \lambda_{k} \\
\text { subject to } & \frac{\lambda_{k}\left|\hat{\mathbf{w}}_{k}^{H} \mathbf{h}_{k}\right|^{2}}{\sum_{j \neq k} \lambda_{j}\left|\hat{\mathbf{w}}_{k}^{H} \mathbf{h}_{j}\right|^{2}+\hat{\mathbf{w}}_{k}^{H} \mathbf{B}_{k} \hat{\mathbf{w}}_{k}} \geq \gamma_{k}
\end{aligned}
$$

where $\hat{\mathbf{w}}_{k} \in \mathbb{C}^{M L \times 1}$ can be interpreted as the receiver beamforming of the dual uplink channel and $\lambda_{k} \geq 0$ has the interpretation of dual uplink power, which is also the Lagrangian dual variable associated with the SINR constraint in (8), and $\mathbf{B}_{k}$ is the dual uplink noise covariance matrix defined as $\mathbf{B}_{k}=\operatorname{diag}\left\{\alpha_{k}^{1} \mathbf{I}_{M}, \alpha_{k}^{2} \mathbf{I}_{M}, \cdots, \alpha_{k}^{L} \mathbf{I}_{M}\right\}, \forall k$.

Proof: We omit the detailed proof since the proof is similar to that in [15].

The optimal solution to (10) is the MMSE receiver [16], which can be readily written as

$$
\hat{\mathbf{w}}_{k}=\left(\sum_{j} \lambda_{j} \mathbf{h}_{j} \mathbf{h}_{j}^{H}+\mathbf{B}_{k}\right)^{-1} \mathbf{h}_{k}
$$

where the dual variable $\lambda_{j}$ is to be determined. In addition, to find the optimal solution $\mathbf{w}_{k}$ to problem (8), we also need to find the scalar $\delta_{k}$ relating $\hat{\mathbf{w}}_{k}$ to $\mathbf{w}_{k}$. Note that it's easy to see that the SINR constraints in both (8) and (10) must be achieved with equality at the optimal point. This observation provides a way to find $\lambda_{j}$, then $\delta_{k}$.

Substituting (11) into the SINR constraint in problem (10) with equality, we can get

$$
\lambda_{k}=\frac{\gamma_{k}}{\mathbf{h}_{k}^{H}\left(\sum_{j \neq k} \lambda_{j} \mathbf{h}_{j} \mathbf{h}_{j}^{H}+\mathbf{B}_{k}\right)^{-1} \mathbf{h}_{k}}
$$

where we use the fact that $\hat{\mathbf{w}}_{k}$ in (11) is colinear with the vector $\left(\sum_{j \neq k} \lambda_{j} \mathbf{h}_{j} \mathbf{h}_{j}^{H}+\mathbf{B}_{k}\right)^{-1} \mathbf{h}_{k}$, which can be easily verified by matrix inversion lemma $[17]^{2}$. The expression in (12) implies that $\lambda_{k}$ can be found numerically by fixed-point method, whose convergence is guaranteed by the fact that the function in (12) is a standard function [19]; see [15], [20].

Now, by substituting $\mathbf{w}_{k}=\sqrt{\delta_{k}} \hat{\mathbf{w}}_{\mathbf{k}}$ into the SINR constraint in (8) with equality, we get $K$ linear equations with $K$ unknowns $\delta_{k}, k=1,2, \cdots, K$ :

$$
\frac{1}{\gamma_{k}} \delta_{k}\left|\hat{\mathbf{w}}_{k}^{H} \mathbf{h}_{k}\right|^{2}=\sum_{j \neq k} \delta_{j}\left|\hat{\mathbf{w}}_{k}^{H} \mathbf{h}_{j}\right|^{2}+\sigma^{2}, \quad \forall k .
$$

Therefore, $\delta_{k}$ can be obtained by solving a system of linear equations:

$$
\boldsymbol{\delta}=\mathbf{F}^{-1} \mathbf{1} \sigma^{2}
$$

where $\boldsymbol{\delta}=\left[\delta_{1}, \delta_{2}, \cdots, \delta_{K}\right], \mathbf{F}$ is defined as: $\mathbf{F}_{i i}=$ $\frac{1}{\gamma_{i}}\left|\hat{\mathbf{w}}_{i}^{H} \mathbf{h}_{i}\right|^{2}$, and $\mathbf{F}_{i j}=-\left|\hat{\mathbf{w}}_{j}^{H} \mathbf{h}_{i}\right|^{2}$ for $i \neq j$, and $\mathbf{1}$ denotes the all-one vector.

We summarize the proposed algorithm in the following:

\footnotetext{
${ }^{2}$ MMSE receiver in general is not unique. The expression in (11) is optimal up to a scalar. See results in [18].
}

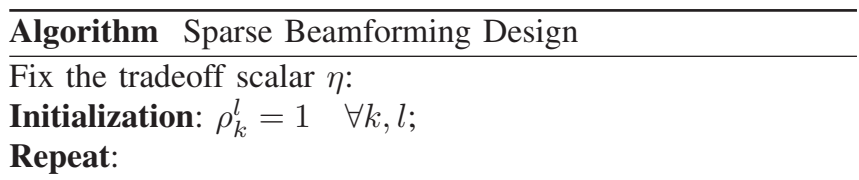

1) Find the optimal dual variable $\lambda_{k}$ according to (12) using fixed-point method;

2) Compute the optimal dual uplink receiver beamforming $\hat{\mathbf{w}}_{k}, \forall k$ according to (11);

3) Update $\mathbf{w}_{k}=\sqrt{\delta_{k}} \hat{\mathbf{w}}_{\mathbf{k}}, \forall k$ with $\delta_{k}$ found by (14);

4) Update $\rho_{k}^{l}$ according to (9).

Until convergence

To find a different tradeoff point between total transmit power and sum backhaul, change $\eta$ and repeat the above steps.

\section{B. Comparison with Algorithms in [8]}

As mentioned earlier, the reweighted $\ell_{1}$-norm approach has already been used to solve the sparse beamforming problem in the previous work [8]. However, [8] uses a formulation which is slightly different from (5); it studies the problem of minimizing the total number of BS-user cooperation links, i.e. $\sum_{k}\left\|\left[\left\|\mathbf{w}_{k}^{1}\right\|_{2},\left\|\mathbf{w}_{k}^{2}\right\|_{2}, \cdots,\left\|\mathbf{w}_{k}^{L}\right\|_{2}\right]\right\|_{0}$, subject to SINR and power constraints ${ }^{3}$. Moreover, [8] expresses the backhaul rate in (4) directly using $\ell_{2}$-norm of the beamforming vectors. After approximating the $\ell_{0}$ norm in (4) by the weighted $\ell_{1}$ norm, the resulting optimization problem becomes an SOCP as follows:

$$
\begin{aligned}
\underset{t_{k}^{l}, \mathbf{w}_{k}^{l}}{\operatorname{minimize}} & \sum_{k, l} \rho_{k}^{l} t_{k}^{l} \\
\text { subject to } & \left\|\mathbf{w}_{k}^{l}\right\|_{2} \leq t_{k}^{l}, \forall k, l \\
& \sum_{k, l}\left(t_{k}^{l}\right)^{2} \leq P \\
& \operatorname{SINR}_{k} \geq \gamma_{k}, \forall k
\end{aligned}
$$

where $P$ denotes the total power budget, and the SINR constraint can also be cast into a SOCP form [20].

The key advantage of the algorithm proposed in this paper as compared to (15) is that (15) can only be solved numerically using, for example, the interior-point method. The complexity of such a general purpose solver is much higher than the uplink-downlink duality approach proposed in this paper.

This SOCP approach is referred to as "Algorithm 1" in [8]. In Algorithm 1, sparse beamforming vectors are obtained by repeatedly solving (15) with iteratively updated weights $\rho_{k}^{l}$. In [8], the authors choose to update the weights according to $\rho_{k}^{l}=\frac{1}{t_{k}^{l}+\epsilon}, \forall k, l$, where $\epsilon$ is some fixed small positive value. Note that this reweigthing function can be seen as a special case of (9) with $p=1 / 2$ and with fixed regularization constant $\epsilon$. Numerically, this paper shows that by adopting an optimized $p$, the performance of Algorithm 1 can be improved.

To address the complexity issue arising from the need to use the interior-point method to solve (15), [8] also proposes

\footnotetext{
${ }^{3}$ In [8], the authors considered per-BS power constraint. For fair comparison with our algorithm, we state the sum power constraint problem here.
} 
an "Algorithm 2", which first solves the following sum power minimization problem

$$
\begin{array}{ll}
\underset{\mathbf{w}_{k}^{l}}{\operatorname{minimize}} & \sum_{k, l}\left\|\mathbf{w}_{k}^{l}\right\|_{2}^{2} \\
\text { subject to } & \operatorname{SINR}_{k} \geq \gamma_{k}, \forall k
\end{array}
$$

then manually removes the BS-user cooperation links corresponding to the least transmit powers until the desired tradeoff point is achieved.

Note that the problem (16) has the same computational complexity as (8), as the uplink-downlink duality approach can be used in both cases. Rather than manually deleting the BS-user cooperation links with the least transmit powers, the algorithm proposed in this paper dynamically selects the BS cluster for each user by iteratively updating the weights in (8). Numerically, this paper shows that the iterative reweighting approach gives superior performance as compared to manual link removal. It is also interesting to point out that Algorithm 2 of [8] can be thought of as a special case of the algorithm proposed in this paper, where all the $\alpha_{k}^{l}$ 's in (8) are set to be 1 , except for the ones corresponding to the least transmit powers, which are set to $+\infty$ in each iteration.

\section{Simulation Results}

The effectiveness of our proposed algorithm is validated through simulations based on a 7-cell $(L=7)$ wrap-around multicell setup with 3 users per cell $(K=21)$ and 4 transmit antennas $(M=4)$ at each BS. The BS-to-BS distance is set at $0.8 \mathrm{~km}$, and the noise power spectral density is $-162 \mathrm{dBm} / \mathrm{Hz}$. The channels from the BSs to the users are generated according to a distance-dependent path-loss model $P L(\mathrm{~dB})=128.1+37.1 \log _{10}(d)$ with $8 \mathrm{~dB} \log$-normal shadowing and a Rayleigh component, where $d$ is the distance between the BS to the user in $\mathrm{km}$. For this setup, numerical experiments show that the proposed algorithm works well with $p=4 / 3$ and $\tau=10^{-10}$ in updating the weight $\rho_{k}^{l}$ in (9).

We first set the tradeoff constant $\eta=0$ (i.e. minimizing the total backhaul only) and set SINR target $\gamma_{k}=15 \mathrm{~dB}$ for every user to illustrate how the sparse beamforming vectors are formed in our proposed algorithm. Fig. 3 shows the transmit power distribution over all 7 BS's for serving user 3 in cell 2 (as shown in the topology in Fig. 2). From Fig. 3, we see that as the iterations progress, BSs 2 and 4 form a serving cluster for the user, while all the other BSs eventually drop their transmit power to zero.

In Figs. 4 and 5, we compare the performance of the proposed algorithm with Algorithms 1 and 2 in [8] in terms of the tradeoff between total transmit power and sum backhaul capacity over all BS's under SINR $=5 \mathrm{~dB}$ and $15 \mathrm{~dB}$ respectively. For the proposed algorithm, we simulate a series of different tradeoff constant $\eta$ 's to get different points along the tradeoff curve. For Algorithm 1, we set different total power budgets $P$, and for each $P$, we solve problem (15) iteratively to find the minimum total number of cooperation links needed, which gives one point on the tradeoff curve. For Algorithm 2, we iteratively remove the BS-user cooperation link corresponding

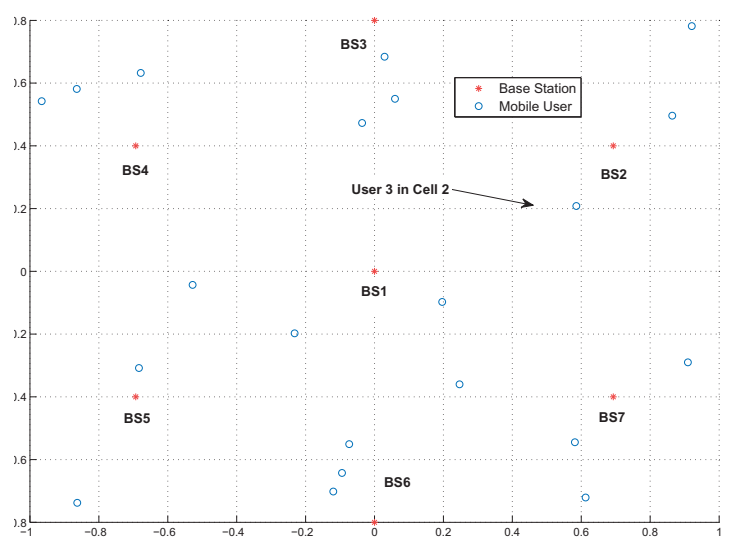

Fig. 2. 7-cell wrap-around topology with 3 users/cell randomly distributed.

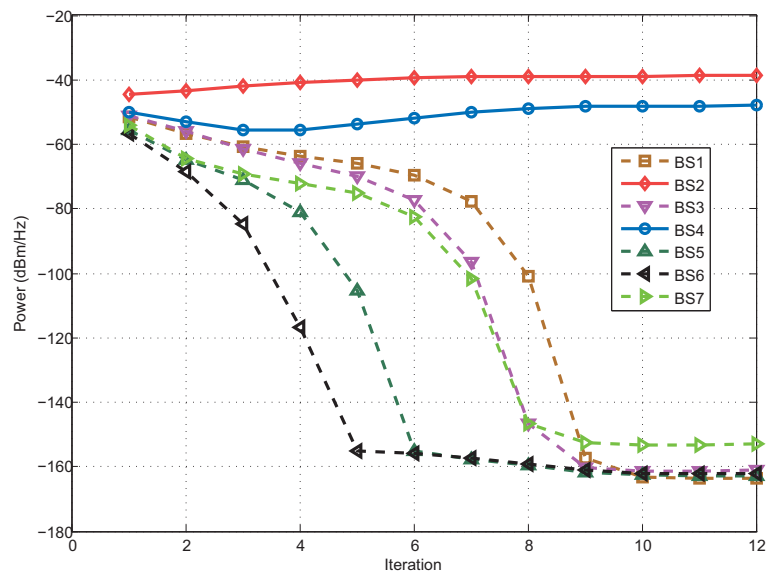

Fig. 3. Transmit powers of the $7 \mathrm{BSs}$ for serving user 3 in cell 2 under $\mathrm{SINR}=15 \mathrm{~dB}$ and $\eta=0$.

to the least transmit power and find the corresponding minimal total transmit power as in (16) until the problem becomes infeasible. Note that in this case since all the users have the same fixed SINR target, optimizing the total backhaul capacity is equivalent to optimizing the total number of cooperation links, as the two are related by a constant $R_{k}=\log (1+\mathrm{SINR})$.

Simulation results show that the proposed algorithm has almost the same performance as Algorithms 1 and 2 of [8] at SINR $=5 \mathrm{~dB}$, whereas at $\mathrm{SINR}=15 \mathrm{~dB}$, the proposed algorithm outperforms the algorithms of [8]. More specifically, as can be seen in Fig. 5, to serve users at the SINR of $15 \mathrm{~dB}$, under a total transmit power of $-14 \mathrm{dBm} / \mathrm{Hz}$ across the $7 \mathrm{BSs}$ (corresponding to about $-23 \mathrm{dBm} / \mathrm{Hz}$ average per-BS transmit power), our proposed algorithm can reduce the total number of cooperation links by 5 as compared to the solution provided by Algorithm 2 of [8]. Likewise, at a typical average cluster size of 3 for each user, which corresponds to a total number of active cooperation links of 63 , our proposed algorithm can reduce the amount of total transmit power by more than $2 \mathrm{~dB}$ as compared with Algorithm 2 of [8]. Note that Algorithm 1 of [8] cannot achieve fewer than 66 cooperation links, which appears to be a significant disadvantage of the SOCP formulation. 


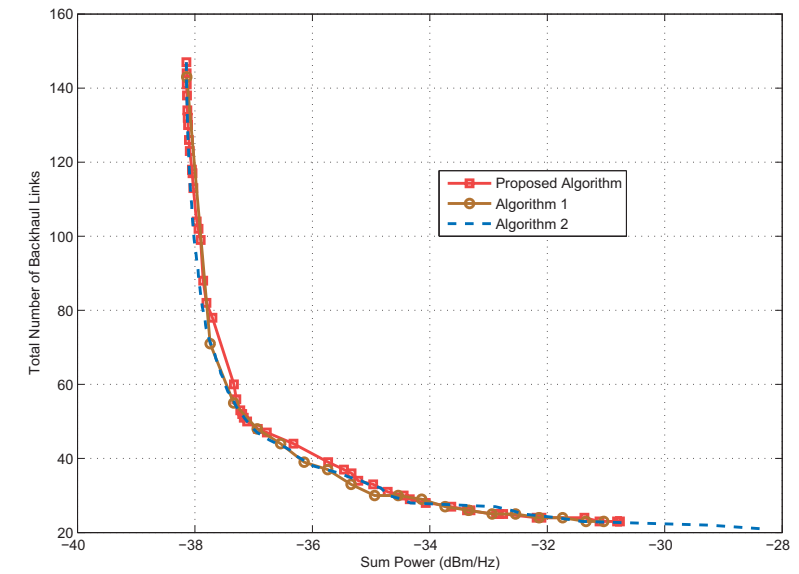

Fig. 4. The tradeoff between the total number of cooperation links and the total transmit power across the $7 \mathrm{BSs}$ under SINR $=5 \mathrm{~dB}$.

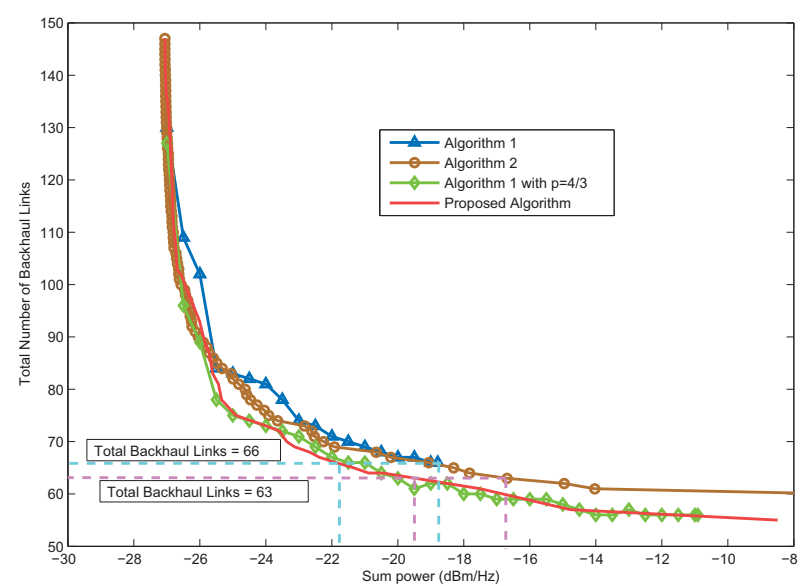

Fig. 5. The tradeoff between the total number of cooperation links and the total transmit power across the $7 \mathrm{BSs}$ under SINR $=15 \mathrm{~dB}$.

To illustrate the effectiveness of the reweighting function adopted in this paper, we also plot the performance of Algorithm 1 of [8] but with weights updated according to (9) for $p=4 / 3$, denoted as 'Algorithm 1 with $\mathrm{p}=4 / 3$ ' in Fig. 5. We see that 'Algorithm 1 with $p=4 / 3$ ' significantly improves the original Algorithm 1, and it now performs approximately the same as our proposed algorithm.

Finally, it should be emphasized that our proposed algorithm is significantly less complex than Algorithm 1 in [8]. Thus, even at the same performance, the proposed algorithm still has considerable advantage.

\section{CONCLUSION}

This paper investigates the tradeoff between total transmit power and sum backhaul capacity over all BSs in a network MIMO system with limited cooperation. By adopting a compressive sensing approach of using reweighted $\ell_{1}$-norm to approximate the $\ell_{0}$-norm, we turn the original nonconvex problem into a series of convex weighted power minimization problem, which can be solved using a low-complex uplinkdownlink duality approach. The proposed algorithm can efficiently find a sparse network-wide beamforming vector for each user where the entries corresponding to the non-serving BSs eventually go down to zero in the iterative process. Simulation results show that the proposed algorithm can achieve a better tradeoff between total transmit power and sum backhaul capacity than existing methods in the high SINR regime.

\section{REFERENCES}

[1] D. Gesbert, S. Hanly, H. Huang, S. Shamai Shitz, O. Simeone, and W. Yu, "Multi-Cell MIMO Cooperative Networks: A New Look at Interference," IEEE J. Sel. Areas Commun., vol. 28, no. 9, pp. 1380 -1408 , Dec. 2010.

[2] S. Venkatesan, A. Lozano, and R. Valenzuela, "Network MIMO: Overcoming Intercell Interference in Indoor Wireless Systems," in Proc. IEEE Forty-First Asilomar Conf. on Signals, Syst. and Comput., Nov. 2007, pp. 83-87.

[3] F. Boccardi and H. Huang, "Limited Downlink Network Coordination in Cellular Networks," in Proc. IEEE Int. Symp. Personal, Indoor and Mobile Radio Commun. (PIMRC), 2007, pp. 1-5.

[4] S. Kaviani, O. Simeone, W. Krzymien, and S. Shamai, "Linear Precoding and Equalization for Network MIMO With Partial Cooperation," IEEE Trans. Veh. Technol., vol. 61, no. 5, pp. 2083-2096, 2012.

[5] E. Candes, M. Wakin, and S. Boyd, "Enhancing Sparsity by Reweighted $\ell_{1}$ Minimization," Journal of Fourier Analysis and Applications, vol. 14, no. 5, pp. 877-905, 2008.

[6] Y. Zeng, E. Gunawan, Y. L. Guan, and J. Liu, "Joint base station selection and linear precoding for cellular networks with multi-cell processing," in IEEE TENCON, 2010, pp. 1976-1981.

[7] J. Zhao and Z. Lei, "Clustering methods for base station cooperation," in Proc. IEEE Wireless Commun. and Networking Conf. (WCNC), 2012.

[8] J. Zhao, T. Q. S. Quek, and Z. Lei, "Coordinated Multipoint Transmission with Limited Backhaul Data Transfer," accepted in IEEE Trans. Wireless Commun., 2013.

[9] S. Boyd and L. Vandenberghe, Convex optimization. Cambridge University Press, 2004.

[10] S.-J. Kim, S. Jain, and G. Giannakis, "Backhaul-constrained multicell cooperation using compressive sensing and spectral clustering," in IEEE Int. Workshop on Signal Process. Advances in Wireless Commun. (SPAWC), 2012, pp. 65-69.

[11] Y.-F. Liu, Y.-H. Dai, and Z.-Q. Luo, "Joint power and admission control via linear programming deflation,” IEEE Trans. Signal Process., vol. 61 , no. 6, pp. 1327-1338, 2013.

[12] M. Hong, R.-Y. Sun, H. Baligh, and Z.-Q. Luo, "Joint Base Station Clustering and Beamformer Design for Partial Coordinated Transmission in Heterogenous Networks," IEEE J. Sel. Areas Commun., vol. 31, no. 2, pp. 226-240, Feb. 2013.

[13] S. Mehryar, A. Chowdhery, and W. Yu, "Dynamic Cooperation Link Selection for Network MIMO Systems with Limited Backhaul Capacity," in Proc. IEEE Int. Conf. Commun. (ICC), 2012.

[14] W. Yu and T. Lan, "Transmitter Optimization for the Multi-Antenna Downlink with Per-Antenna Power Constraints," IEEE Trans. Signal Process., vol. 55, no. 6, pp. 2646-2660, 2007.

[15] H. Dahrouj and W. Yu, "Coordinated beamforming for the multicell multi-antenna wireless system," IEEE Trans. Wireless Commun., vol. 9, no. 5, pp. 1748-1759, 2010.

[16] T. Kailath, A. H. Sayed, and B. Hassibi, Linear estimation. Prentice Hall NJ:, 2000, vol. 1.

[17] D. Tylavsky and G. Sohie, "Generalization of the Matrix Inversion Lemma," in Proc. IEEE, vol. 74, no. 7, 1986, pp. 1050-1052.

[18] F. Yuan and C. Yang, "Equivalence of SLNR Precoder and ZF Precoder in Downlink MU-MIMO Systems," [online] arXiv:1202.1888, 2012.

[19] R. D. Yates, "A Framework for Uplink Power Control in Cellular Radio Systems,” IEEE J. Sel. Areas Commun., vol. 13, no. 7, pp. 1341-1347, 1995.

[20] A. Wiesel, Y. C. Eldar, and S. Shamai, "Linear precoding via conic optimization for fixed mimo receivers," IEEE Trans. Signal Process. vol. 54, no. 1, pp. 161-176, 2006. 